\title{
A coprecipitation technique to prepare $\mathrm{NaNbO}_{3}$ and $\mathrm{NaTaO}_{3}$
}

\author{
V SAMUEL ${ }^{\dagger \dagger}$, A B GAIKWAD ${ }^{\dagger}$ and V RAVI* \\ Physical and Materials Chemistry Division, ${ }^{\dagger}$ Centre for Materials Characterization, ${ }^{\dagger \dagger}$ Catalysis Division, \\ National Chemical Laboratory, Pune 411 008, India
}

MS received 18 October 2005; revised 8 December 2005

\begin{abstract}
A simple coprecipitation technique has been used successfully for the preparation of pure, ultrafine, single phases of $\mathrm{NaNbO}_{3}(\mathrm{NN})$ and $\mathrm{NaTaO}_{3}(\mathrm{NT})$. An alcoholic solution of ammonium carbonate and ammonium hydroxide was used to precipitate $\mathrm{Na}^{+}$and $\mathrm{Nb}^{5+}$ (or $\mathrm{Ta}^{5+}$ ) cations under basic conditions as carbonate and hydroxide, respectively. On heating at $700^{\circ} \mathrm{C}$, these precursors produce respective products. For comparison, both NN and NT powders were also prepared by the traditional solid state method. The phase purity and lattice parameters were studied by powder X-ray diffraction (XRD). The particle size and morphology were studied by scanning electron microscopy (SEM).
\end{abstract}

Keywords. Ceramics; oxides; chemical synthesis; X-ray methods; electronic materials; electron microscopy.

\section{Introduction}

Alkali metal niobates and tantalates $\left(\mathrm{LiNbO}_{3}\right.$ and $\left.\mathrm{LiTaO}_{3}\right)$ have excellent electro-optical and photorefractive properties and find extensive applications as optical wave guides and modulators and surface acoustic wave devices, e.g. $\mathrm{NaNbO}_{3}$ is reported (Raveski and Prosandeev 2002; Aydi et al 2004; Castro et al 2004) to be a promising antiferroelectric material for lead free piezoelectric applications. $\mathrm{NaTaO}_{3}$ has also been investigated (Istomin et al 2002; Kato and Kudo 2003) for possible application as a photocatalyst for splitting of water into $\mathrm{H}_{2}$ and $\mathrm{O}_{2}$. Traditionally these materials were prepared by solid state reaction which leads to inhomogeneity in composition and coarse particles. Chemical methods, e.g. co-precipitation, sol-gel, hydrothermal and colloid emulsion techniques, allow to efficiently control the morphology and chemical composition of the prepared powders. Sol-gel using alkoxides, hydrothermal and colloid emulsions are time consuming and involve highly unstable alkoxides and difficult to maintain reaction conditions. Co-precipitation is one of the more successful wet-chemical techniques for synthesizing ultrafine ceramic powders having narrow particle size distribution (Dhage et al 2003; Gaikwad et al 2004, $2005 \mathrm{a}, \mathrm{b})$. The purpose of this study was to prepare ultrafine $\mathrm{NaNbO}_{3}$ and $\mathrm{NaTaO}_{3}$ powders using the co-precipitation technique from simple water soluble inorganic salts. This process can avoid complex steps such as refluxing of alkoxides, resulting in less time consumption compared to other techniques. The limitation of the co-

\footnotetext{
*Author for correspondence (ravi@ems.ncl.res.in)
}

precipitation process is that cations should have similar solubility product.

\section{Experimental}

For preparing $\mathrm{NaNbO}_{3}$ and $\mathrm{NaTaO}_{3}$, niobium $(\mathrm{V})$ oxide, tantalum(V) oxide, sodium hydroxide, ethyl alcohol, ammonium carbonate and standard ammonia solution were used and all the starting materials were of $\mathrm{AR}$ grade (Loba chemie). A stoichiometric amount of $\mathrm{NaOH}$ was dissolved in distilled water $(100 \mathrm{ml})$ and required quantity of $\mathrm{Nb}_{2} \mathrm{O}_{5}$ or $\mathrm{Ta}_{2} \mathrm{O}_{5}$ was dissolved in $\mathrm{HF}$ after heating in a hot water bath for $10 \mathrm{~h}$. Then an equal amount of ethyl alcohol was added to the above solution mixture containing both sodium hydroxide and niobium (or tantalum) fluoride. Then, aqueous solution of ammonium carbonate and ammonium hydroxide was added with constant stirring to the above solution mixture to reach $\mathrm{pH} \sim 9$ to ensure complete precipitation of sodium carbonate and niobium (or tantalum) hydroxide. The precipitate was filtered, washed several times with distilled water to remove anions and dried in an oven at $100^{\circ} \mathrm{C}$ for $12 \mathrm{~h}$. The oven dried powders were calcined at various temperatures ranging from 200 $800^{\circ} \mathrm{C}$ for $12 \mathrm{~h}$. For comparison, both NN and NT samples were also prepared by the ceramic powder processing method from stoichiometric mixtures of oxides or carbonates which were mixed, ground several times and heated at $900^{\circ} \mathrm{C}$ for $12 \mathrm{~h}$. The powder X-ray pattern was recorded for all the samples by using a Philips PW-1710 model $\mathrm{X}$-ray diffractometer and $\mathrm{CuK}_{\alpha}$ radiation. For lattice parameter and interplanar distance $(d)$ calculation, the samples were scanned in the $2 \theta$ range of $10-80^{\circ}$ for a period of $5 \mathrm{~s}$ in the step scan mode. Silicon was used as an inter- 
nal standard. Least squares method was used to determine the lattice parameters. SEM observations were made using a Leica Cambridge 440 microscope. All powders were dispersed in amyl acetate.

\section{Results and discussion}

The precursor obtained has been calcined at $700^{\circ} \mathrm{C}$ for $6 \mathrm{~h}$. Figures 1 and 2 show the XRD pattern of the calcined powders indicating formation of pure $\mathrm{NaNbO}_{3}$ and $\mathrm{Na}$ $\mathrm{TaO}_{3}$. The observed $d$-lines pattern (crystal structure is orthorhombic) for $\mathrm{NaNbO}_{3}$ is similar to that reported in the literature (JCPDS 14-603). The calculated lattice parameters by least squares fit are $a=5 \cdot 511 \AA, b=5 \cdot 557 \AA$ and $c=15 \cdot 540 \AA$. The compound, $\mathrm{NaTaO}_{3}$, has cubic structure (JCPDS 2-873). The calculated lattice parameter

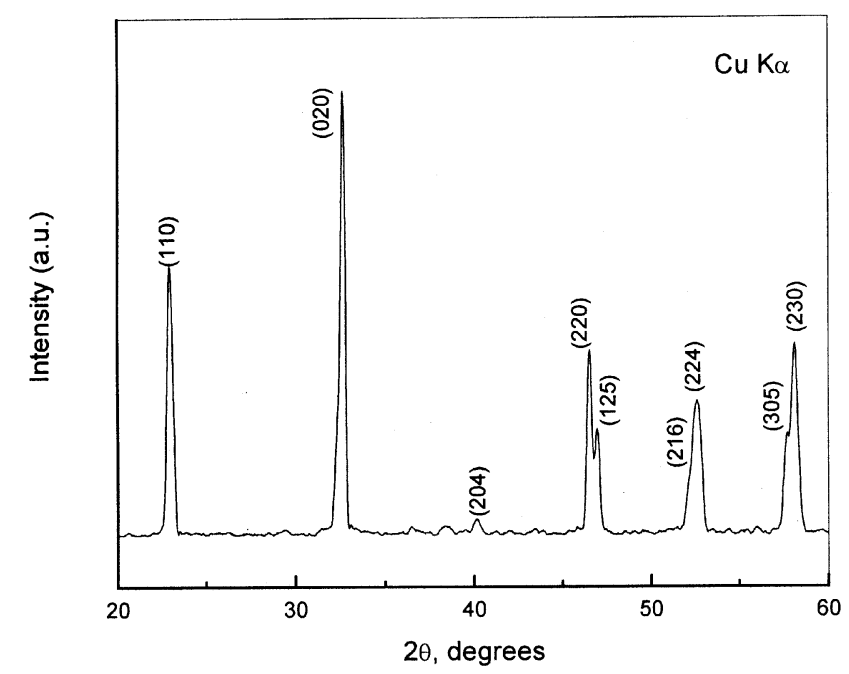

Figure 1. $\mathrm{XRD}$ of $\mathrm{NN}$ powder calcined at $700^{\circ} \mathrm{C}$.

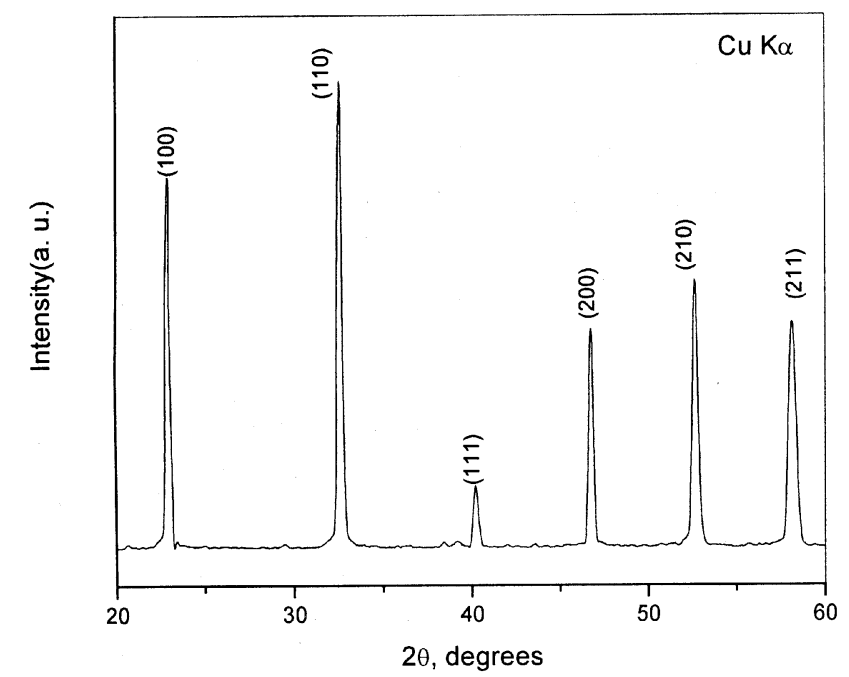

Figure 2. XRD of NT powder calcined at $700^{\circ} \mathrm{C}$. by least squares fit is $a=3 \cdot 88 \AA$. The conventional solid state method also produces NN and NT phases at $900^{\circ} \mathrm{C}$ with a comparatively larger particle size of $\sim 1 \mu \mathrm{m}$. The particle size and morphology of the calcined powders were examined by scanning electron microscopy. Particle morphology of calcined powder $\left(700^{\circ} \mathrm{C}\right.$ for $\left.6 \mathrm{~h}\right)$ prepared by the coprecipitation process was irregular in shape, with an average primary particle size of around $200 \mathrm{~nm}$ (figures 3 and 4). The average particle size of $150 \mathrm{~nm}$ was calculated from Scherrer's formula

$$
t=K \ddot{e} / B \cos \theta_{\mathrm{B}}
$$

where $t$ is the average size of the particles assuming particles to be spherical, $K=0 \cdot 9, \lambda$ is the wavelength of X-ray radiation, $B$ the full width at half maximum of the di-

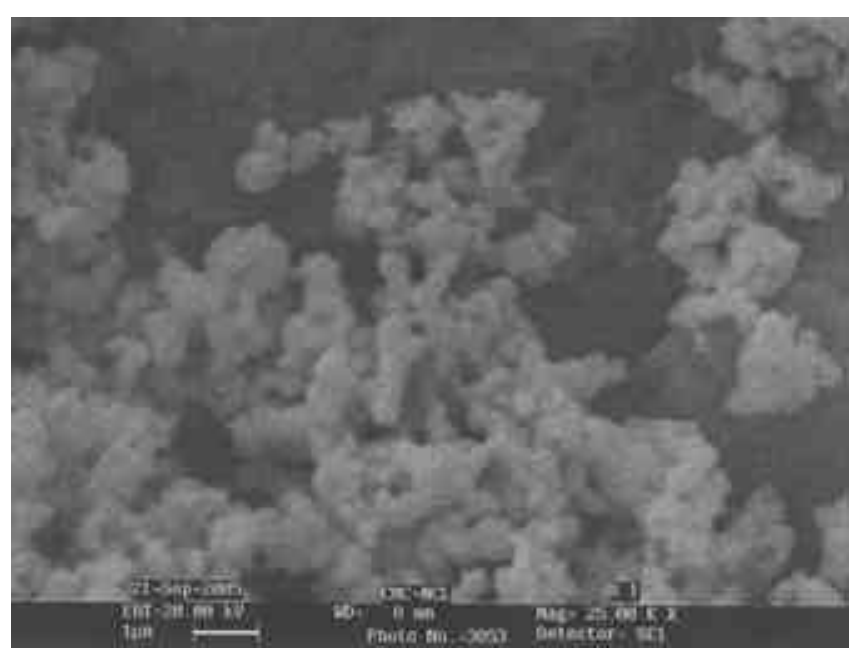

Figure 3. SEM of NN powder calcined at $700^{\circ} \mathrm{C}$.

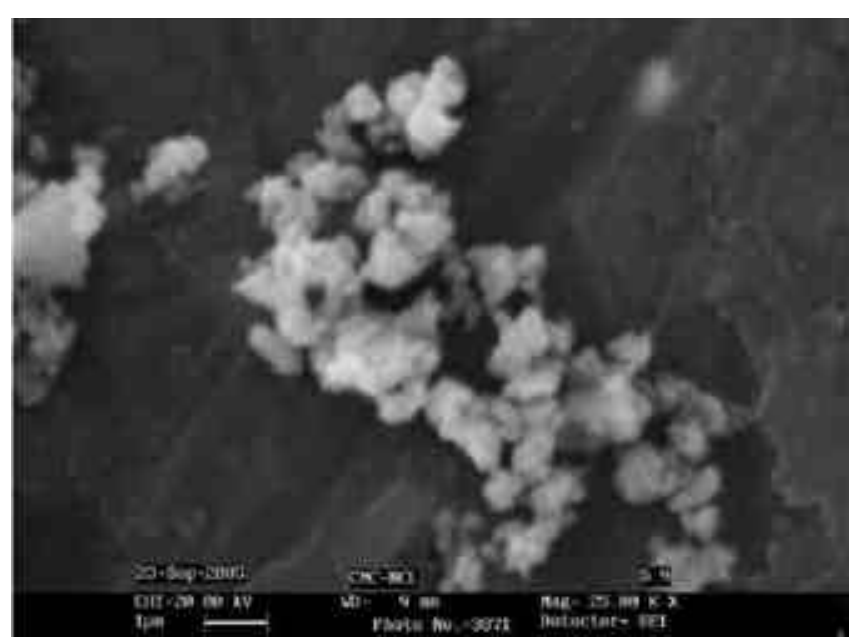

Figure 4. SEM of NT powder calcined at $700^{\circ} \mathrm{C}$. 
ffracted peak (after correcting for instrumental broadening) and $\theta_{\mathrm{B}}$ the angle of diffraction.

\section{Conclusions}

A simple coprecipitation method was used to prepare ultrafine particles of $\mathrm{NaNbO}_{3}$ and $\mathrm{NaTaO}_{3}$. Both $\mathrm{NN}$ and NT phases were crystallized at $700^{\circ} \mathrm{C}$ with average particle size of $200 \mathrm{~nm}$.

\section{Acknowledgement}

One of the authors (VR) acknowledges DST, Govt. of India (grant no. SP/S1/H-19/2000) for financial assistance.

\section{References}

Aydi A, Khemakhem H, Boudaya C, Muhll R V and Simon A 2004 Solid State Sci. 6333

Castro A, Jimenez B, Hungria T, Moure A and Pardo L 2004 J. Euro. Ceram. Soc. 24941

Dhage S R, Ravi V and Date S K 2003 Bull. Mater. Sci. 26215

Gaikwad S P, Dhesphande S B, Khollam Y, Samuel V and Ravi V 2004 Mater. Lett. 583474

Gaikwad S P, Potdar H S, Samuel V and Ravi V 2005a Ceram. Int. 31379

Gaikwad S P, Dhage S R and Ravi V 2005b J. Mater. Sci. Mater. Electron. 16229

Istomin S Y, Svensson G and Kohler J 2002 Solid State Sci. 4191

Kato H and Kudo A 2003 Catalysis Today 78561

Raveski I P and Prosandeev S A 2002 J. Phys. \& Chem. Solids 631939 